\title{
EFICÁCIA DA ESCOLHA DO PROTETOR AUDITIVO PEQUENO, MÉDIO E GRANDE EM PROGRAMA DE CONSERVAÇÃO AUDITIVA
}

\section{Efficacy in the choice of small, medium or large auricular protector under auditive conservation program}

\author{
Marleide Aparecida Griggio Rodrigues (1), Adriana Adilia Dezan (2), \\ Luciana Lozza de Moraes Marchiori ${ }^{(3)}$
}

\begin{abstract}
RESUMO
Objetivo: verificar a eficácia da escolha do protetor auditivo por tamanhos Pequeno, Médio, Grande em um programa de conservação auditiva. Método: fizeram parte do estudo 30 indivíduos com audição normal previamente avaliados, de 18 a 45 anos de idade, sendo 15 do gênero feminino e 15 do gênero masculino, de Indústria Moveleira. Os sujeitos foram submetidos à avaliação audiológica convencional, audiometria em campo livre sem protetor auricular de inserção, com protetor tamanho universal e com tamanho adequado a cada indivíduo. Para análise estatística foi aplicado teste T Paramétrico para análise das diferenças. Resultados: $O$ teste $T$ Paramétrico pareado, mostrou significância, na comparação dos protetores auriculares na freqüência de $4 \mathrm{KHz}$. Conclusão: os resultados mostraram eficácia dos protetores auriculares pequeno e grande sobre os protetores tamanho universal em um programa de conservação auditiva.
\end{abstract}

DESCRITORES: Perda Auditiva Provocada por Ruído; Ruído Ocupacional; Audição

\section{INTRODUÇÃO}

A busca constante do homem por conforto e comodidade promove um crescimento industrial acelerado e desordenado, acarretando a invenção de máquinas, brinquedos e veículos cada vez mais barulhentos, tornando os ambientes ruidosos e insalubres. Desde 2500 anos atrás, a humanidade conhece os efeitos prejudiciais do ruído à saúde, através de textos relatando a surdez dos trabalhadores que viviam próximo às cataratas do rio Nilo no antigo Egito. Sabe-se que o ruído é inimigo silencioso e sorrateiro e atinge o homem sob vários aspectos. Pessoas

(1) Fonoaudióloga, Audioprev, Especialização em Audiologia Clínica.

(2) Fonoaudióloga, Clínica Interata, Especialização em Motricidade Oral.

(3) Fonoaudióloga, Docente nos cursos de Fonoaudiologia e Farmácia da Universidade Norte do Paraná, Doutora em Medicina e Ciências da Saúde pela Universidade Estadual de Londrina. aparentemente com boa saúde podem estar sendo vítima da nocividade do ruído ${ }^{1}$.

Estatisticamente, estima-se que $15 \%$ da população exposta a ruído constante de $90 \mathrm{~dB}$, oito horas por dia, durante cinco dias por semana e 50 semanas por ano, apresentarão lesão auditiva após dez anos. Segundo a Organização Mundial do Trabalho, há mais de 140 milhões de pessoas expostas a níveis perigosos de ruído ocupacional no mundo. Pela Organização Mundial de Saúde a perda auditiva induzida por ruído (PAIR) seria hoje a causa de perda auditiva mais evitável no mundo ${ }^{2}$.

A proteção, na grande maioria dos casos, é simples. Basta que sejam utilizados protetores auriculares. A prova disto é que, se simplesmente colocarmos os dedos nos ouvidos, como boa vedação, já será possíve I a redução significativa da sensação do ruído ${ }^{2}$.

O funcionamento de um protetor auditivo depende, porém, de suas características e das características fisiológicas e anatômicas do usuário.

O primeiro protetor de inserção de ouvido foi criado por Dr. Cousins que, em 1884, Plessner da Alemanha, patenteou um plug esférico para encai- 
xar-se dentro do canal auditivo, e lembrou-se pela primeira vez a necessidade de diferentes tamanhos de protetores. Variações destas formas foram descritas nos anos seguintes: na segunda guerra mundial estimulou a pesquisa de desenvolvimento de protetores de inserção. Avaliações sistemáticas de vários designs de plugs foram feitas pelo Comitê Americano de defesa de pesquisa ${ }^{2}$.

Os resultados preliminares resultaram no conhecido v-51v onde foi feito originalmente em três tamanhos. Como sua eficácia só foi comprovado em $83 \%$ dos adultos masculinos, foi então introduzido os tamanhos extra-pequeno e extra-grande. Houve várias tentativas para produzir um protetor de colocação universal ${ }^{3}$.

Em 1961 Santi projetou protetor tamanho universal. Hoje existem vários tipos, marcas e modelos de protetores auditivos com cerca de 1000 modelos em nível internacional. No Brasil os mais utilizados dos modelos Plug são os de tamanho universal ${ }^{3}$.

Constatou-se que mesmo com todas as tecnologias utilizadas, e todas as pesquisas realizadas, ainda hoje encontramos certa dificuldade na obtenção do protetor adequado, pois como já mencionado anteriormente, o ser humano tem sua característica fisiológica e anatômica própria, e por isso muitas vezes tem-se problemas para obter uma boa vedação de protetores auditivos dos modelos plug de tamanho universal encontrados no mercado ${ }^{4}$.

$\mathrm{Na}$ prática clínica, pode-se perceber que os protetores auditivos de tamanho universal usados no mercado não se adequam a todos os trabaIhadores, principalmente quando a adaptação é feita em mulheres. Às vezes ficam grandes demais, outras vezes pequenos, sendo necessário à utilização de tamanhos diversificados. Por outro lado, há escassez de trabalhos que demonstrem a eficácia na escolha de protetores auditivo por tamanho Pequeno (P), Médio (M), Grande (G), em um programa de conservação auditiva.

A partir das colocações acima citadas visou-se verificar a eficácia da escolha do protetor auditivo por tamanhos P,M,G em um programa de conservação auditiva.

\section{MÉTODOS}

A população estudada consistiu de 30 indivíduos adultos, entre 18 e 45 anos, sendo 15 do gênero feminino e 15 do gênero masculino de Empresa Moveleira.

Como critérios de inclusão considerou-se audiometria preliminar normal bilateralmente; idade de 18 a 45 anos; e experiência na utilização de protetor auditivo.
Foram previamente excluídos da pesquisa os indivíduos que apresentaram rolha de cerúmen e/ou queixa de zumbido.

Os procedimentos consistiram em Audiometria tonal convencional em cabine acusticamente tratada; Audiometria em campo livre sem protetor auditivo; Audiometria em campo livre com protetor tamanho universal; Medição por fonoaudióloga para verificação do tamanho do conduto auditivo utilizando os próprios protetores auriculares P.M.G, uma vez que estes são mais eficazes que - Otômetro para a citada verificação, e indicação do protetor adequado da marca POMP®, única empresa que oferece atualmente protetores de tamanhos variados, P.M.G.; Audiometria em campo livre com o protetor adequado.

Os exames foram realizados individualmente, utilizando o audiômetro $A D 27$ Interacoustics e áudio em campo livre, nas freqüências $500,1000,2000,3000,4000,6000$ e 8000 Hertz $(\mathrm{Hz})$.

Esta pesquisa foi autorizada pelo Comitê de Ética e Pesquisa do Centro de Especialização em Fonaoudiologia Clínica, sob o no 161/05.

Os resultados foram apresentados na forma descritiva com as médias dos resultados obtidos nas audiometrias em campo livre, com o protetor tamanho Universal e com os tamanhos P e G. Foi aplicado o teste $T$ de Student paramétrico pareado, para comparação dos protetores auriculares, considerando neste caso o índice de significância em $5 \%(p \leq 0.05)$.

\section{RESULTADOS}

Os resultados encontrados, conforme demonstra a Tabela 1 mostram que houve melhora significativa na comparação das médias das freqüências testadas de 500 à $8000 \mathrm{hz}$, do protetor adequado em comparação ao protetor tamanho universal/M. Das 30 orelhas testadas, $43.3 \%$ se adequaram ao tamanho diferenciado P ou G, 23.3\% apesar de não obterem diferença nos limiares $500-8 \mathrm{Khz}$, mas se referiram a maior conforto com o tamanho escolhido como consta na Tabela 1.

A Tabela 2 compara a melhora da atenuação dos protetores nas freqüências independentes de 500 a $8000 \mathrm{~Hz}$. Percebe-se que a melhora significativa $(p<0,05)$ aconteceu na freqüência de $4000 \mathrm{hz}$, sendo esta uma das freqüências mais acometidas na Perda Auditiva Induzida por Níveis de Pressão Sonora (PAINPS). 
Tabela 1 - Distribuição das diferenças nos limiares 500-8Khz em decibéis das audiometrias realizadas em campo livre, com protetor tamanho universal, tamanho P e tamanho G

\begin{tabular}{|c|c|c|c|c|}
\hline & $\begin{array}{l}\text { Áudio em Campo } \\
\text { Livre }\end{array}$ & $\begin{array}{l}\text { Audio c/ protetor } \\
\text { tamanhoU/M }\end{array}$ & $\begin{array}{l}\text { Audio com } \\
\text { protetor tamanho } \\
\text { P }\end{array}$ & $\begin{array}{l}\text { Audio com } \\
\text { protetor tamanho } \\
\text { G }\end{array}$ \\
\hline Paciente & X 500-8Khz & X 500-8Khz & X 500-8Khz & X500-8Khz \\
\hline (2) & 15,71 & 39,29 & 43,5714 & \\
\hline 2 & 17,86 & 36,43 & 44,2857 & \\
\hline 3 & 22,86 & 39,29 & 49,2857 & \\
\hline 4 & 20,00 & 47,14 & & \\
\hline 5 & 16,43 & 41,43 & & \\
\hline 6 & 21,43 & 44,29 & 45,7143 & \\
\hline 7 & 15,00 & 38,57 & & \\
\hline 8 & $-2,14$ & 22,14 & & 25,714285 \\
\hline 9 & 16,43 & 50,00 & & 51,428571 \\
\hline 10 & 3,57 & 7,86 & & 13,571429 \\
\hline 11 & 10,71 & 31,43 & & 26,428571 \\
\hline 12 & 0,00 & 12,86 & & 13,571429 \\
\hline 13 & 1,43 & 2,86 & & 15 \\
\hline 14 & 7,14 & 20,00 & & 14,285714 \\
\hline 15 & 3,57 & 30,71 & 26,4286 & \\
\hline 16 & 5,71 & 20,71 & 19,2857 & \\
\hline 17 & 10,71 & 40,71 & & \\
\hline 18 & 10,71 & 40,71 & & \\
\hline 19 & 2,86 & 15,71 & 20 & \\
\hline 20 & 1,43 & 15,71 & 3,57143 & \\
\hline 21 & 1,43 & 8,57 & 10,7143 & \\
\hline 22 & 7,14 & 17,14 & & \\
\hline 23 & 15,00 & 26,43 & & 30 \\
\hline 24 & 7,86 & 27,86 & & 21,428571 \\
\hline 25 & $-0,71$ & 15,00 & & \\
\hline 26 & 4,29 & 22,86 & & \\
\hline 27 & 3,57 & 12,86 & & \\
\hline 28 & 5,71 & 19,29 & & \\
\hline 29 & 0,00 & 15,71 & 10 & \\
\hline 30 & 18,57 & 46,43 & 51,428571 & \\
\hline
\end{tabular}

OBS: Das 30 orelhas testadas, $43.3 \%$ se adequaram ao tamanho diferenciado $\mathrm{P}$ ou G, $23.3 \%$ apesar de não obterem diferença nos limiares $500-8 \mathrm{Khz}$, mas se referiram a maior conforto com o tamanho escolhido

c/ - com; Hz- Hertz, U/M - Universal; P- Pequeno, G - Grande

Tabela 2- Distribuição dos p-valores referente à aplicação do Teste T Paramétrico de Student para variáveis dependentes em relação à melhora da atenuação dos protetores nas freqüências independentes de $500 \mathrm{a} 8000 \mathrm{~Hz}$

\begin{tabular}{llllllll}
\hline & $\mathrm{D} 1-500$ & $\mathrm{D} 1-1000$ & $\mathrm{D} 1-2000$ & $\mathrm{D} 1-3000$ & $\mathrm{D} 1-4000$ & $\mathrm{D} 1-6000$ & $\mathrm{D} 1-8000$ \\
\hline $\mathrm{D} 2-500$ & 0,315073 & 0,366533 & 0,155366 & 0,064269 & $0,010075^{*}$ & 0,406186 & $0,011632^{*}$ \\
D2-1000 & 0,334162 & 0,211732 & 0,325124 & 0,187922 & $0,022436^{*}$ & 0,608279 & $0,022795^{*}$ \\
D2-2000 & $0,010196^{*}$ & $0,002213^{*}$ & 0,409389 & 0,348658 & $0,000052^{*}$ & 0,253353 & 0,357995 \\
D2-3000 & $0,025786^{*}$ & $0,006755^{*}$ & 0,76405 & 0,529632 & $0,000149^{*}$ & 0,341187 & 0,180281 \\
D2-4000 & 0,917691 & 0,822586 & 0,071235 & $0,012633^{*}$ & $0,028603^{*}$ & 0,07438 & $0,00024^{*}$ \\
D2-6000 & $0,047874^{*}$ & $0,018103^{*}$ & 0,648717 & 0,499522 & $0,001587^{*}$ & 0,234195 & 0,357995 \\
D2-8000 & $0,027295^{*}$ & $0,016369^{*}$ & 0,494296 & 0,291804 & $0,000651^{*}$ & 0,196965 & 0,498924 \\
\hline
\end{tabular}

* diferença significativa $p<0,05$

D1-com protetor adequado, D2-com outro protetor, Hz- Hertz 


\section{DISCUSSÃO}

A perda auditiva induzida por ruído (PAIR) é doença ocupacional de alta prevalência nos países industrializados, destacando-se como um dos agravos à saúde do trabalhador mais prevalentes nas indústrias brasileiras ${ }^{5}$.

Embora a PAIR tenha atingido proporções praticamente endêmicas no meio industrial, estudos científicos sobre a sua história natural nos trabalhadores brasileiros ainda são escassos ${ }^{6}$. Destaca-se também a grande imprecisão na quantificação do nível de exposição individual ao ruído, observada nas indústrias brasileiras ${ }^{6}$.

Toivonen ${ }^{7}$ questiona a atenuação do ruído referida pelo fabricante para os protetores auriculares, principalmente se não forem utilizados adequadamente (colocação, prazo de trocas e condições de higiene).

Vários estudos têm sido realizados sobre a eficácia do protetor auricular na audiologia ocupacional, desde que o Comitê Nacional de Ruído e Conservação Auditiva lançou no Brasil o Boletim n. $6^{8}$, as diretrizes básicas de um Programa de Conservação da Audição (PCA), com recomendações mínimas para a sua elaboração, contendo as seguintes etapas: reconhecimento e avaliação de riscos para a audição, gerenciamento audiométrico, medidas de proteção coletivas, medidas de proteção individual, educação e motivação, gerenciamento de dados e avaliação do programa ${ }^{6}$, porém há inexistência de estudos sobre a importância do tamanho P,M,G, na eficácia da proteção do ruído ocupacional. Além disso, a utilização de protetores auriculares enquanto medida principal no controle dos efeitos do ruído não tem se mostrado suficiente para evitar o agravamento da PAIR ${ }^{6}$.

Todo protetor, seja concha, abafadores, plugues de inserção, atenua o ruído criando uma barreira para reduzir o som que chega por via aérea à membrana timpânica porém o nível de proteção obtido depende do grau de vedação do protetor, de forma que qualquer vazamento permite que o som passe pelo protetor.

O Otômetro ou medidor auricular ${ }^{9}$ é o aparelho mais indicado para medir o conduto auditivo segundo a literatura da área, no entanto, nesta pesquisa, se pode constatar que os funcionários souberam diferenciar com maior precisão qual o protetor mais adequado através da utilização dos próprios protetores auditivos. Isto, provavelmente devido aos mesmos serem mais flexíveis e indicarem com melhor precisão o tamanho correto do protetor a ser utilizado.

Utilizou-se para a pesquisa funcionários com experiência no uso de protetores auditivos e mesmo assim em alguns casos observou-se que havia dife- renças nas médias dos limiares auditivos testados, de acordo com a colocação dos mesmos.

Por esse fator não só a escolha do tamanho correto é importante, mas há necessidade de um adequado treinamento de como utilizá-los, pois como é visto na literatura ${ }^{6}$, as ações consideradas como sendo de preservação da audição dos trabalhadores são predominantemente baseadas na realização de audiometrias e no fornecimento de protetores auriculares. No entanto há necessidade de uma melhor investigação sobre como os protetores auriculares estão senso utilizados (atenuação real, colocação, higiene etc. $)^{6}$.

Como é visto na literatura ${ }^{10}$, o desempenho acústico do protetor auditivo envolve, não apenas as características físicas do protetor e sua relação com o ouvido, mas também as limitações anatômicas e fisiológicas do usuário, sendo que para a escolha do protetor auditivo deve ser feito um trabalho individual, no qual sejam considerados aspectos de atenuação inerentes ao protetor (qualidade), características pessoais do usuário (tamanho do meato acústico externo, formato do rosto e da cabeça, compatibilidade com outros equipamentos de proteção individual-EM, tipo de atividade, utilização adequada, preferências) e nível de ruído no qual o operário trabalha. Dessa forma, o protetor auditivo precisa deixar de ser escolhido pela empresa de forma indiscriminada, e passar a ser escolhido considerando o usuário obedecendo a aspectos técnicos. Alguns aspectos devem nortear a definição do protetor, como o fato dos protetores auditivos possuírem características que devem ser consideradas no momento da sua seleção e escolha, sendo elas a atenuação oferecida, o conforto, o tamanho do protetor, a facilidade de colocação, a compatibilidade com o usuário e com outros equipamentos e a preferência pessoal do usuário ${ }^{11}$. Esses aspectos puderam ser observados em parte na presente pesquisa, especialmente no que se refere à importância do tamanho adequado de cada protetor auricular para a real atenuação do ruído.

A partir deste estudo percebe-se que novas pesquisas devem ser feitas nesta área, uma vez que a perda auditiva relacionada ao trabalho, particularmente a perda auditiva induzida por ruído (PAIR), é doença ocupacional de alta prevalência nos países industrializados, destacando-se como um dos agravos à saúde do trabalhador mais prevalentes nas indústrias brasileiras ${ }^{12}$ e que somente a utilização de protetores auriculares não é o suficiente no controle dos efeitos do ruído para prevenção da PAIR, sendo a escolha do tamanho adequado de cada protetor auricular, de grande importância na implantação de um PCA nas empresas. 
Salientando-se que os cuidados em relação à audição certamente servirão para evitar as frustrações pela diminuição da capacidade de compreender a linguagem oral, ocasionadas pela diminuição da acuidade auditiva ${ }^{13,14}$, passível de ser instalada em indivíduos expostos ao ruído ocupacional.

\section{CONCLUSÃO}

Os resultados mostraram eficácia dos protetores auriculares pequeno e grande sobre os protetores tamanho universal em um programa de conservação auditiva.

\begin{abstract}
Purpose: to check the efficacy in the choice of small, medium or large auricular protector under auditive conservation program. Methods: 30 individuals, between 18 to 45 -year old, with normal hearing, were part of the study previously evaluated: 15 women and 15 men, all working in a furniture industry. The individuals were submitted to the conventional audiological evaluation, audiometry in free field without auricular insertion protector, with universal size protector and with size adequate to each individual. For statistical analysis we used Parametric T test for analyzing the differences. Results: the Parametric $T$ test showed significance, during the comparison of the auricular protectors in the frequency of $4 \mathrm{KHz}$. Conclusion: these results demonstrate efficacy in choice of small, medium or large auditive protector, under auditive conservation program.
\end{abstract}

KEYWORDS: Hearing Loss, Noise-Induced; Noise, Occupational; Hearing

\section{REFERÊNCIAS}

1.Nudelman A, Costa A, Seligman J, Ibáñez RN. Pair: perda auditiva induzida pelo ruído. Porto Alegre: Bagaggem; 1997. p. 261-85.

2.Regazzi RD, Araújo GM. Perícia e avaliação de ruído e calor passo a passo: teoria e prática. Rio de Janeiro: Impresso do Brasil; 2002.

3. Gerges SNY. Protetores auditivos. Florianópolis: NR; p. 29-35.

4.Musiek FE, Rintelmann WF. Perspectivas atuais em avaliação auditiva. Barueri: Manoel; 2001. p. 461-74. 5. Almeida SIC, Albernaz PLM, Zaia PA, Xavier OG, Karazawa EHI. História natural da perda auditiva ocupacional provocada por ruído. Rev Assoc Med Bras. 2000; 46(2):143-58.

RECEBIDO EM: 27/09/06

ACEITO EM: 17/11/06

Endereço para correspondência:

Rua Belo Horizonte, 1399 / 401

Londrina - PR

CEP: 86020-060

E-mail: luciana.marchiori@unopar.br
6. Guerra MR, Lourenço PMC, Texeira MTB, Alves MJM. Prevalência de perda auditiva induzida por ruído em empresa metalúrgica. Rev Saúde Publ. 2005; 39:527-30.

7. Toivonen M, Paakkonen R, Savolainen S, Lehtomaki K. Noise attenuation and proper insertion of earplugs into ear canals. Ann Occup Hyg. 2002; 46(6):527-30. 8. Comitê Nacional de Ruído e Conservação Auditiva. Boletim ํㅡ. 6. Arquivos da Fundação Otorrinolaringologia 2000; 4(2) (periódico on line:http://www.hcnet.usp.br/ otorrino/arq42/com.htm)

9. Superintendência de Controle de Endemias do Estado de São Paulo. Index de saúde do trabalhador. Disponível em: URL: www.sucen.sp.gov.br/ saude_trabalhador

10. Recomendações mínimas para a elaboração de um PCA. São Paulo: Comitê Nacional de Ruído e Conservação Auditiva; 1999.

11. Avagliano A, Almeida K. Estudo do desempenho de diferentes tipos de protetores auditivos. Rev CEFAC. 2001; 3(1):77-87.

12. Almeida SIC, Albernaz PLM, Zaia PA, Xavier OG, Karazawa EHI. História natural da perda auditiva ocupacional provocada por ruído. Rev Assoc Med Bras. 2000; 46(2):143-58.

13. Tambs K. Moderate effects of hearing loss on mental health and subjective well-being: results from the nord-trondelag hearing loss study. Psychosom Med. 2004; 66(5):776-82.

14. Cox RM, Alexander GC, Gray GA. Who wants a hearing aid? Personality profiles of hearing aid seekers. Ear Hear. 2005; 26(1):12-26. 